\title{
High-Temperature Fractional Quantum Hall States
}

\author{
Evelyn Tang, ${ }^{1}$ Jia-Wei Mei, ${ }^{1,2}$ and Xiao-Gang Wen ${ }^{1}$ \\ ${ }^{1}$ Department of Physics, Massachusetts Institute of Technology, Cambridge, Massachusetts 02139, USA \\ ${ }^{2}$ Institute for Advanced Study, Tsinghua University, Beijing, 100084, People's Republic of China
}

(Received 14 December 2010; published 6 June 2011)

\begin{abstract}
We show that a suitable combination of geometric frustration, ferromagnetism, and spin-orbit interactions can give rise to nearly flatbands with a large band gap and nonzero Chern number. Partial filling of the flatband can give rise to fractional quantum Hall states at high temperatures (maybe even room temperature). While the identification of material candidates with suitable parameters remains open, our work indicates intriguing directions for exploration and synthesis.
\end{abstract}

DOI: 10.1103/PhysRevLett.106.236802

PACS numbers: 73.43.Cd, 05.30.Fk

Landau symmetry breaking $[1,2]$ has been the standard theoretical concept in the classification of phases and transitions between them. However, this theory turned out to be insufficient when the fractional quantum Hall $(\mathrm{FQH})$ state $[3,4]$ was discovered. These highly entangled quantum states are not distinguished by symmetries but characterized by new topological quantum numbers such as robust ground state degeneracy [5,6] and robust nonAbelian Berry's phases [7] of the ground states [8]. The new kind of order revealed in these topological quantum numbers is named topological order [8,9]. Recently, it was realized that topological order can be interpreted as patterns of long-range quantum entanglement [10-12]. This entanglement has important applications for topological quantum computation: the robust ground state degeneracy can be used as quantum memory [13]. Fractional defects from the entangled states which carry fractional charges [4] and fractional statistics [14-16] (or non-Abelian statistics $[17,18])$ can be used to perform fault tolerant quantum computation $[19,20]$.

Presently, highly entangled gapped phases in FQH systems $[3,4]$ are only realized at very low temperatures. Here we present a proposal to realize these states at high temperatures (even room temperature). The ideal is to combine spin-orbit coupling, ferromagnetism, and geometric frustration. Both spin-orbit coupling and ferromagnetism can have high energy scales and can appear at room temperature. In some cases, combining them leads to energy bands with nonzero Chern numbers and filling such an energy band will give rise to integer quantum Hall states. Further, in geometrically frustrated systems-lattices on which hopping is frustrated-some of these topologically nontrivial energy bands can be very flat $[21,22]$. These would mimic Landau levels in free space. When such a flatband with a nonzero Chern number is partially filled (such as $1 / 3$ or $1 / 2$ filled), FQH states can appear. Here we study a simple example of this idea on the geometrically frustrated kagome lattice.

Several aspects of these ideas have been active in recent research. Spin-orbit coupling can lead to a topological insulator in various geometrically frustrated systems [23-26], and noncollinear magnetic order can lead to integer quantum Hall states [24,27]. Alternatively, interactions in geometrically frustrated systems can break time-reversal symmetry [28-36] which again can give rise to integer quantum Hall states. Here we show that an extension of these ideas may set the stage for FQH states and other highly entangled states with fractional statistics and fractional charges-possibly even at room temperature.

Nearly flatband; nonzero Chern number-We consider nearest and next-nearest-neighbor hopping on a kagome lattice with spin-orbit interactions in the Hamiltonian

$$
\begin{aligned}
H= & -t_{1} \sum_{\langle i j\rangle \sigma} c_{i \sigma}^{\dagger} c_{j \sigma}+i \lambda_{1} \sum_{\langle i j\rangle \alpha \beta}\left(\mathbf{E}_{i j} \times \mathbf{R}_{i j}\right) \cdot \boldsymbol{\sigma}_{\alpha \beta} c_{i \alpha}^{\dagger} c_{j \beta} \\
& -t_{2} \sum_{\langle\langle i j\rangle\rangle \sigma} c_{i \sigma}^{\dagger} c_{j \sigma}+i \lambda_{2} \sum_{\langle\langle i j\rangle\rangle \alpha \beta}\left(\mathbf{E}_{i j} \times \mathbf{R}_{i j}\right) \cdot \boldsymbol{\sigma}_{\alpha \beta} c_{i \alpha}^{\dagger} c_{j \beta},
\end{aligned}
$$

where $c_{i \sigma}^{\dagger}$ creates an electron with spin $\sigma$ on site $\mathbf{r}_{i}$ (see Fig. 1). Here $\langle i j\rangle$ denotes nearest neighbors and $\langle\langle i j\rangle\rangle$ nextnearest neighbors. The second and fourth terms are time-reversal invariant and describe spin-orbit interactions. $\mathbf{R}_{i j}$ is the distance vector between sites $i$ and $j$ and $\mathbf{E}_{i j}$ the electric field from neighboring ions experienced along $\mathbf{R}_{i j}$.

To obtain FQH states we need to break time-reversal symmetry. This is likely to happen spontaneously from exchange effects in the flatband that cause ferromagnetism [28-30]. Alternatively, one can apply an external magnetic field or couple the system to a ferromagnet. In the extreme limit the electron spins are totally polarized within the partially filled band-the case we examine here. Hence, we consider spin-orbit coupling that also conserves $S_{z}$; i.e., the electric field on each site is in the $2 \mathrm{D}$ plane. First studying just nearest-neighbor hopping $\left(t_{2}=\lambda_{2}=0\right)$, in momentum-space Eq. (1) is 


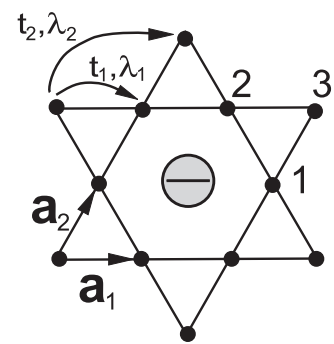

FIG. 1. The kagome lattice is a triangular Bravais lattice with a 3 -point basis labeled $l=1,2,3 ; \mathbf{a}_{1}=\hat{x}$ and $\mathbf{a}_{2}=(\hat{x}+\sqrt{3} \hat{y}) / 2$ are the basis vectors. In the metallic kagome lattice $\mathrm{Fe}_{3} \mathrm{Sn}_{2}$, spinorbit coupling arises from the electric field due to the $\mathrm{Sn}$ ion at the center of the hexagon.

$$
\begin{aligned}
H_{\mathbf{k}}= & -2 t_{1}\left(\begin{array}{ccc}
0 & \cos k_{1} & \cos k_{2} \\
\cos k_{1} & 0 & \cos k_{3} \\
\cos k_{2} & \cos k_{3} & 0
\end{array}\right) \\
& \pm i 2 \lambda_{1}\left(\begin{array}{ccc}
0 & \cos k_{1} & -\cos k_{2} \\
-\cos k_{1} & 0 & \cos k_{3} \\
\cos k_{2} & -\cos k_{3} & 0
\end{array}\right),
\end{aligned}
$$

where $\mathbf{a}_{1}=\hat{x}, \quad \mathbf{a}_{2}=(\hat{x}+\sqrt{3} \hat{y}) / 2, \quad \mathbf{a}_{3}=\mathbf{a}_{2}-\mathbf{a}_{1}$, and $k_{n}=\mathbf{k} \cdot \mathbf{a}_{n}$. We use units where the hopping parameter $t_{1}=1$. The $+(-)$ sign refers to spin up (down) electrons; from here we focus on just the spin up electrons.

The spectrum consists of three energy bands and is gapless at $\lambda_{1}=0, \pm \sqrt{3}$. At all other points the spectrum is gapped and the top and bottom bands have unit Chern number with opposite sign while the middle band has zero Chern number. The Chern number is defined as [37]

$$
c=\frac{1}{2 \pi} \int_{B Z} d^{2} k F_{12}(k)
$$

where $F_{12}(k)$ is the associated field strength given by $F_{12}(k)=\frac{\partial}{\partial k_{1}} A_{2}(k)-\frac{\partial}{\partial k_{2}} A_{1}(k)$ with the Berry connection $A_{\mu}(k)=-i\left\langle n_{\mathbf{k}}\left|\frac{\partial}{\partial k_{\mu}}\right| n_{\mathbf{k}}\right\rangle$. In the above $\left|n_{\mathbf{k}}\right\rangle$ is a normalized wave function of the respective band.

Focusing on the lowest band which has a nonzero Chern number, we look for where this band is very flat compared to the band gap and the energy scale of interactions. We denote $W$ as the maximum bandwidth of the lowest band, $\Delta_{12}$ as the minimum band gap between the two lowest bands, and $U$ as the strength of electron-electron interactions. When $U \gg W$, interaction effects dominate kinetic energy and partially filling the flatband would favor the Laughlin state [4]. Since band mixing could destroy band flatness, ideally $\Delta_{12} \gg U$. Hence we aim to maximize the ratio $\Delta_{12} / W$ in order to obtain $\mathrm{FQH}$ states. As the middle band has zero Chern number, any mixing between only two of the bands would not change the Chern number of the lowest band. If the lowest band remains flat even with mixing then $\Delta_{13}$, the minimum band gap between the lowest and highest bands (and consequently the ratio $\left.\Delta_{13} / W\right)$ is also of interest.

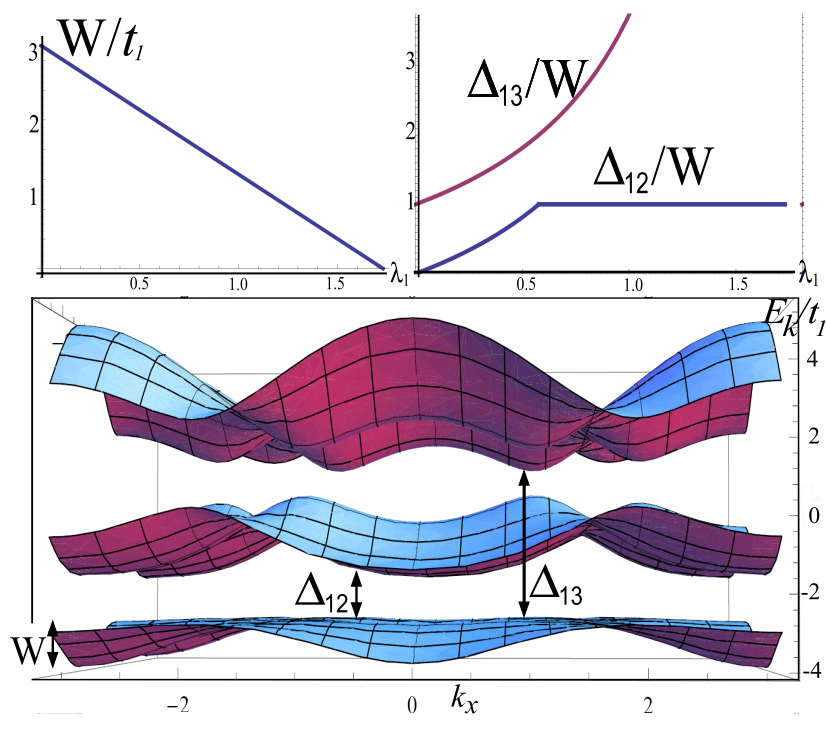

FIG. 2 (color online). Results for nearest-neighbor hopping as a function of $\lambda_{1}$ (nearest-neighbor spin-orbit coupling). The bandwidth of the lowest band $W$ vanishes at $\lambda_{1}=\sqrt{3}$; however, $W \geq \Delta_{12}$ always where $\Delta_{12}$ is the band gap between the two lowest bands. Here we show the band structure for $\lambda_{1}=1$ where $W=1.3$ and $\Delta_{13} / W=3.7$. The spectrum does not have a clear separation of energy scales between $\Delta_{12}, W$ and $U$, the interaction strength, which could be due to limitations of the nearestneighbor hopping model.

We find that $W \geq \Delta_{12}$ always - as the bandwidth vanishes so does the band gap between the two lower bands (topological symmetry in real space [38]), see Fig. 2. Here we show the band structure for $\lambda_{1}=1$ where $W=1.3$ and $\Delta_{13} / W=3.7$. As $\Delta_{12} / W \leq 1$ always, the spectrum does not have a clear separation of energy scales. When interactions are on the order of $W$, the bands will mix. This scenario is quite different from Landau levels in free space that are flat and well separated, which could be due to limitations of this simplest model.

For a more realistic scenario we include second-nearestneighbor hopping which adds terms in the Hamiltonian

$$
\begin{aligned}
& H_{\mathbf{k}}=-2 t_{2}\left(\begin{array}{ccc}
0 & \cos \left(k_{2}+k_{3}\right) & \cos \left(k_{3}-k_{1}\right) \\
0 & \cos \left(k_{1}+k_{2}\right) \\
0 & 0
\end{array}\right) \\
& +i 2 \lambda_{2}\left(\begin{array}{ccc}
0 & -\cos \left(k_{2}+k_{3}\right) & \cos \left(k_{3}-k_{1}\right) \\
0 & -\cos \left(k_{1}+k_{2}\right) \\
0 & 0
\end{array}\right) \text {. }
\end{aligned}
$$

In this larger parameter space the band maxima and minima are no longer fixed at the same symmetry points. We find that the largest values of $\Delta_{12} / W$ (and $\Delta_{13} / W$ ) occur when $\lambda_{1}$ and $\lambda_{2}$ are of the same sign-in which case the results are symmetric under changing signs of both $\lambda$ 's in this spin polarized case. In Fig. $3, \Delta_{12} / W$ is plotted as a function of $t_{2}$ for three values of $\lambda_{1}=\lambda_{2}$. We see that at 


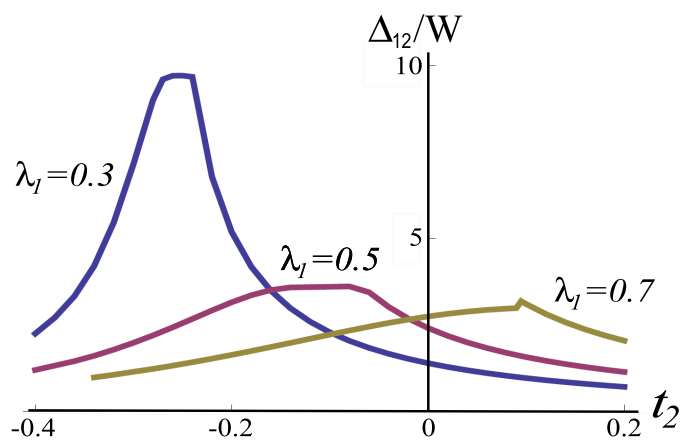

FIG. 3 (color online). With the inclusion of next-nearestneighbor hopping, we obtain much higher band gap to bandwidth ratios. We choose three values of $\lambda_{1}=\lambda_{2}=0.3,0.5$, and 0.7 , and sweep $\Delta_{12} / W$ with $t_{2}$. For lower values of spin-orbit coupling, the ratio peaks at negative $t_{2}$; for relatively higher values of spin-orbit coupling the converse is true.

negative values of $t_{2}$ a lower spin-orbit coupling is needed, while for positive values of $t_{2}$ higher values of spin-orbit coupling would maximize the band gap to bandwidth ratio.

We present two examples where $\Delta_{12} / W$ and $\Delta_{13} / W$ reach high values at $t_{2}=-0.3$. In case 1 , setting $t_{2}=$ $-0.3, \lambda_{1}=0.28$, and $\lambda_{2}=0.2$, we obtain a very flat lowest band separated from the two higher bands by a large gap (see Fig. 4). The values of $\Delta_{12} / W$ and $\Delta_{13} / W$ are 52 and 99, respectively. In another example, all three bands are fairly flat (particularly the lowest one) and mutually well separated. The parameters used are $t_{2}=-0.3, \lambda_{1}=$ 0.6 , and $\lambda_{2}=0$. In case 2 , we obtain $\Delta_{12} / W=8.7$ and $\Delta_{13} / W=24$, respectively (see Fig. 5).

Calculating the Chern number $c$ of the lowest flatband in these two cases, we find it is 1 . This is expected as slowly turning off $t_{2}$ and $\lambda_{2}$ does not close the band gap-and we have previously seen that in the absence of next-nearestneighbor hopping, the lowest band always has unit Chern number. When $\Delta \gg U \gg W$ is satisfied, partial filling of this flatband would favor the FQH state.

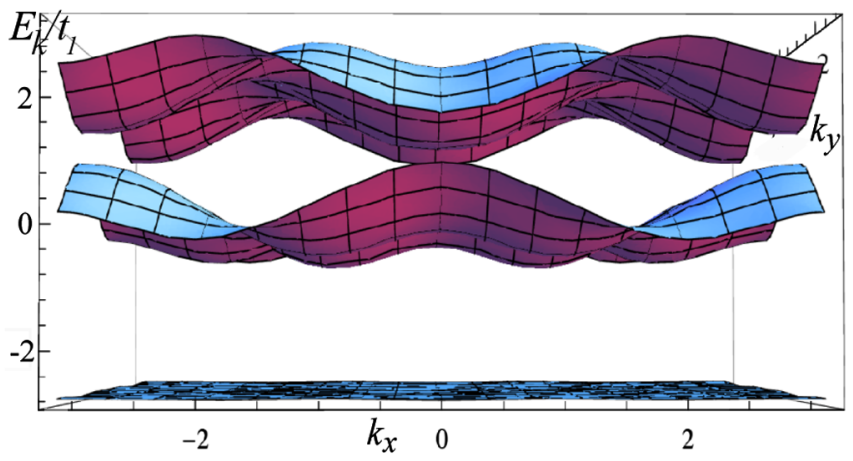

FIG. 4 (color online). A very flat lowest band-well-separated from the two higher bands - is obtained with the parameters $t_{2}=$ $-0.3, \lambda_{1}=0.28$, and $\lambda_{2}=0.2$ (case 1 ). The band gap to bandwidth ratios are high: $\Delta_{12} / W=52$ and $\Delta_{13} / W=99$, respectively.

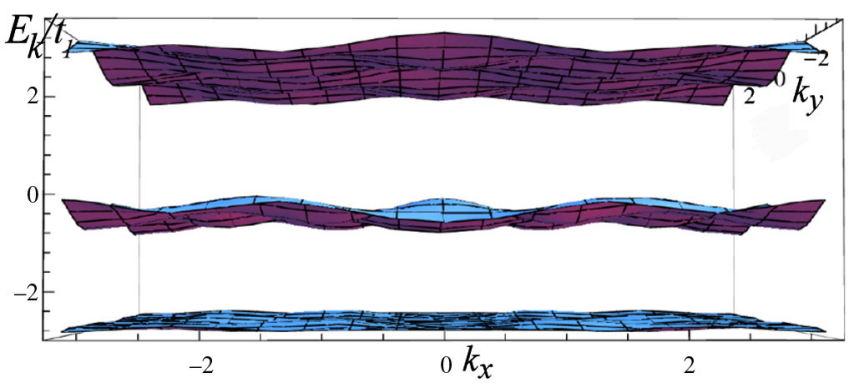

FIG. 5 (color online). Three fairly flatbands are mutually well separated with band gap to bandwidth ratios of $\Delta_{12} / W=8.7$ and $\Delta_{12} / W=24$. Parameters used are $t_{2}=-0.3, \lambda_{1}=0.6$, and $\lambda_{2}=0$ (case 2).

The distribution of the field strength $F_{12}(k)$ in the Brillouin zone is plotted in Fig. 6. We observe there are no singularities or very sharp features but $F_{12}(k)$ varies fairly smoothly especially in the first case with the flatter band. The presence of singularities-e.g., localized at the Dirac point—would have signaled a new (and much larger) length scale in the system. In our case, both the magnetic length scale (arising from spin-orbit interactions) and the variation of field strength $F_{12}(k)$ are on the order of the lattice constant $a$.

Thus, the interaction energy scale is generated from the lattice constant $a: U \sim e^{2} / \varepsilon a$ where $\epsilon$ is the dielectric constant. For a $1 / 3$ filled flatband, analogous to results from FQH states in semiconductor-based systems [39], the gap for this $\nu=1 / 3$ state is roughly $0.09 e^{2} / \varepsilon a=500 \mathrm{~K}$. (We choose $\varepsilon=3$ and $a=10 \AA ; a$ is defined as the square root of the unit cell area.) As the interaction energy scale is a hundred times larger than in semiconductors, we may see the FQH effect at room temperature. As band gaps $\Delta_{12}$ and $\Delta_{13}$ are easily much higher than room temperature, a fully filled band could give the integer quantum Hall state at unusually high temperatures too.

Materials realization.-We see that a suitable combination of geometric frustration, ferromagnetism, and spinorbit interactions can give rise to nearly flatbands with a large band gap and nonzero Chern number. The ferromag-

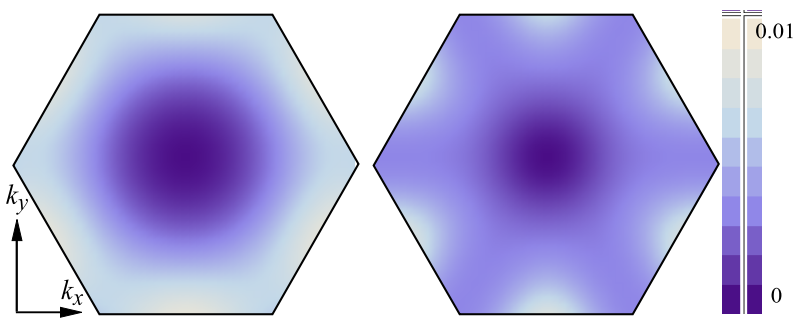

FIG. 6 (color online). Distribution of the field strength $F_{12}(k)$ [Eq. (3)] in the Brillouin zone for the flatbands in cases 1 and 2 discussed above. They do not contain sharp features-especially case 1 with the flatter band-hence the magnetic length scale remains on the order of the lattice constant $a$. 
netism can arise from an external magnetic field, a ferromagnetic substrate for a thin film sample, and/or exchange effects. If the flatband is close to the Fermi energy, partial filling of the flatband can be controlled by doping and give rise to $\mathrm{FQH}$ states at high temperatures.

The choice of parameters in our calculations is based on known values of spin-orbit coupling. For example, in Herbertsmithite (a common copper-based 2D kagome lattice) the spin-orbit interaction is $8 \%$ of the kinetic energy [40]. Other compounds with $4 d$ or $5 d$ orbitals (instead of $3 d$ as in $\mathrm{Cu}$ ) may experience a larger spin-orbit interaction. For instance, the strength of spin-orbit coupling in iridiumbased kagome compounds can be on the order of magnitude of the kinetic energy; hence, the substitution with $4 d$ or $5 d$ atoms in metallic kagome lattices could result in hopping parameters similar to the ones used in our work. Alternatively, making thin films of frustrated lattices with $4 d$ or $5 d$ atoms may lead to a flatband with strong spinorbit coupling, where exchange effects in this flatband could cause ferromagnetism.

Most existing kagome compounds are $\mathrm{Cu}$-based insulators. Some 2D kagome lattices show metallic behavior, for instance $\mathrm{Fe}_{3} \mathrm{Sn}_{2}$ [41,42] which shows ferromagnetism along the $c$ axis above $60 \mathrm{~K}$ and in the kagome plane below $60 \mathrm{~K}$. Also, as spin-orbit interactions can be simulated in cold atom systems $[43,44]$, it is possible to realize our hopping model in such systems. This would provide a method to obtain FQH states in cold atom systems.

In short, flatbands with nonzero Chern number arise in the examples we have given and in other geometrically frustrated systems with suitable levels of spin-orbit interaction. By partially filling these bands, e.g., via doping, one can expect the emergence of $\mathrm{FQH}$ states at high temperatures. While the identification of exact material candidates remains open, our work indicates intriguing directions for synthesis and development.

This research is supported by NSF Grant No. DMR1005541.

Note added.-Recently, we learned that Neupert et al. [45] also discussed the possibility of the $\mathrm{FQH}$ effect in interacting two-band lattice systems, while Sun et al. [46] found flatbands with nonzero Chern numbers on various lattices (including the kagome lattice) after including some complex hopping.

[1] L. D. Landau, Phys. Z. Sowjetunion 11, 26 (1937).

[2] L.D. Landau and E. M. Lifschitz, Statistical Physics (Pergamon, London, 1958).

[3] D. C. Tsui, H. L. Stormer, and A. C. Gossard, Phys. Rev. Lett. 48, 1559 (1982).

[4] R. B. Laughlin, Phys. Rev. Lett. 50, 1395 (1983).

[5] X.-G. Wen, Phys. Rev. B 40, 7387 (1989).

[6] X.-G. Wen and Q. Niu, Phys. Rev. B 41, 9377 (1990).
[7] F. Wilczek and A. Zee, Phys. Rev. Lett. 52, 2111 (1984).

[8] X.-G. Wen, Int. J. Mod. Phys. B 4, 239 (1990).

[9] X.-G. Wen, Adv. Phys. 44, 405 (1995).

[10] A. Kitaev and J. Preskill, Phys. Rev. Lett. 96, 110404 (2006).

[11] M. Levin and X.-G. Wen, Phys. Rev. Lett. 96, 110405 (2006).

[12] X. Chen, Z.-C. Gu, and X.-G. Wen, Phys. Rev. B 82, 155138 (2010).

[13] E. Dennis et al., J. Math. Phys. (N.Y.) 43, 4452 (2002).

[14] J. M. Leinaas and J. Myrheim, Nuovo Cimento B 37, 1 (1977).

[15] F. Wilczek, Phys. Rev. Lett. 49, 957 (1982).

[16] D. Arovas, J. R. Schrieer, and F. Wilczek, Phys. Rev. Lett. 53, 722 (1984).

[17] G. Moore and N. Read, Nucl. Phys. B360, 362 (1991).

[18] X.-G. Wen, Phys. Rev. Lett. 66, 802 (1991).

[19] A. Y. Kitaev, Ann. Phys. (N.Y.) 303, 2 (2003).

[20] C. Nayak et al., Rev. Mod. Phys. 80, 1083 (2008).

[21] H. Katsura et al., Europhys. Lett. 91, 57007 (2010).

[22] D. Green, L. Santos, and C. Chamon, Phys. Rev. B 82, 075104 (2010).

[23] G. Liu et al., Phys. Rev. B 79, 035323 (2009).

[24] H.-M. Guo and M. Franz, Phys. Rev. B 80, 113102 (2009).

[25] H.-M. Guo and M. Franz, Phys. Rev. Lett. 103, 206805 (2009).

[26] Z. Wang and P. Zhang, arXiv:0909.2465.

[27] K. Ohgushi, S. Murakami, and N. Nagaosa, Phys. Rev. B 62, R6065 (2000).

[28] T. Kimura et al., Phys. Rev. B 65, 081307 (2002).

[29] A. Tanaka and H. Ueda, Phys. Rev. Lett. 90, 067204 (2003).

[30] F. Pollmann, P. Fulde, and K. Shtengel, Phys. Rev. Lett. 100, 136404 (2008).

[31] J. Wen et al., Phys. Rev. B 82, 075125 (2010).

[32] S. Rachel and K. L. Hur, Phys. Rev. B 82, 075106 (2010).

[33] N. Hao, P. Zhang, and Y. Wang, arXiv:1009.6068.

[34] Q. Liu, H. Yao, and T. Ma, Phys. Rev. B 82, 045102 (2010).

[35] S. Nishimoto et al., Phys. Rev. Lett. 104, 196401 (2010).

[36] I. Martin and C. D. Batista, Phys. Rev. Lett. 101, 156402 (2008).

[37] D. J. Thouless et al., Phys. Rev. Lett. 49, 405 (1982).

[38] D. L. Bergman, C. Wu, and L. Balents, Phys. Rev. B 78, 125104 (2008).

[39] R. L. Willett et al., Phys. Rev. B 37, 8476 (1988).

[40] A. Zorko et al., Phys. Rev. Lett. 101, 026405 (2008).

[41] L. A. Fenner, A. A. Dee, and A. S. Wills, J. Phys. Condens. Matter 21, 452202 (2009).

[42] T. Kida et al., arXiv:0911.0289.

[43] Y.-J. Lin et al., Nature (London) 462, 628 (2009).

[44] N. Goldman et al., Phys. Rev. Lett. 105, 255302 (2010).

[45] T. Neupert, L. Santos, C. Chamon, and C. Mudry, this issue, Phys. Rev. Lett. 106, 236804 (2011).

[46] K. Sun, Z. Gu, H. Katsura, and S. Das Sarma, following Letter, Phys. Rev. Lett. 106, 236803 (2011). 